Published in final edited form as:

J Hypertens. 2018 February ; 36(2): 234-236. doi:10.1097/HJH.0000000000001576.

\title{
Blood pressure targets in the elderly
}

\author{
Gemma Currie and Christian Delles \\ Institute of Cardiovascular and Medical Sciences, University of Glasgow, Glasgow, UK
}

\begin{abstract}
Several factors surrounding the treatment of hypertension have continually caused controversy over the last decades and remain unresolved. Key examples of these issues include: choice of first-line antihypertensive therapy; the role of combination therapy; blood pressure in the context of other cardiovascular risk factors; and the role of assessment of subclinical and overt organ damage in defining treatment strategies. Nothing, however, has stimulated debate to the extent seen over blood pressure targets.
\end{abstract}

Contemporary guidelines generally advocate lower blood pressure targets compared to earlier recommendations when hypertension was first recognised as an important yet modifiable cardiovascular risk factor. The issue is further illustrated by more sophisticated classification of blood pressure not only in the hypertensive range but also within normotensive values; concepts such as "pre-hypertension" and "high normal blood pressure" illustrate that there could be an optimal blood pressure range that is lower than the current diagnostic threshold of 140/90 mmHg. Discussions about J-shaped and U-shaped curves that describe the relationship between blood pressure and cardiovascular risk are important in this context and have been revisited many times over the years. It has indeed been recognised that comorbidities such as diabetes and renal failure may mandate different and often lower blood pressure targets compared to the general population as a result of altered relationships between blood pressure and cardiovascular risk in these high-risk patients [1]. Studies that specifically address the question of optimal blood pressure targets such as HOT [2] and more recently SPRINT [3] have therefore attracted widespread attention but also critique [4]. It is probably fair to say that there is still debate on optimal blood pressure targets but that a "the lower the better" concept is generally accepted for most patients.

It therefore came as a surprise to the hypertension community when the Eighth Joint National Committee (JNC8) defined a higher blood pressure target for people above the age of 60 years [5]. Of course the decision to recommend a target of 150/90 $\mathrm{mmHg}$ was well justified by the Committee and based on the existing literature, applying a new and rigorous pipeline to examine the existing evidence, in keeping with Institute of Medicine recommendations. However, the $150 / 90 \mathrm{mmHg}$ target in the elderly was still a decision against the general "the lower the better" trend. And it was not accepted by all Committee members; opponents of this target published a "minority report" outlining their views and interpretation of the evidence in more detail compared to the statements in the JNC8 report [6].

Address for Correspondence: Prof. Christian Delles, Institute of Cardiovascular and Medical Sciences, BHF Glasgow Cardiovascular Research Centre, University of Glasgow, 126 University Place, Glasgow, G12 8TA, Scotland, UK; Phone: +44 (141) 330 2749, Fax: +44 (141) 330 3360, Christian.Delles@ glasgow.ac.uk. 
There are good reasons to define different blood pressure targets depending on comorbidities, organ damage, sex and age to take specific pathophysiological mechanisms and cardiovascular risk in different groups of patients into account. In the elderly it has repeatedly been argued that low blood pressure could lead to dizziness and orthostatic reactions thereby increasing the risk of falls and fractures [7]. It has also been argued that increased vascular stiffness in the elderly which results in lower diastolic pressure and higher pulse pressure would require different blood pressure-lowering strategies compared to younger patients [8]. In addition, one of the largest blood pressure trials available at the time of the JNC8 process, the Hypertension in the Very Elderly Trial (HYVET), specifically looked into a 150/80 $\mathrm{mmHg}$ target [9]. Nevertheless, there have been concerns that a more relaxed blood pressure target in the elderly would lead to undertreatment of those who may benefit most from antihypertensive therapy and that JNC8 has sent out a "wrong" message to the clinical community [6].

This discussion about $10 \mathrm{mmHg}$ in the elderly is of course only a continuation of the general debate on blood pressure targets that we have witnessed for many years. There will be no absolute "right" or "wrong" and it is well possible that this recommendation, not unlike other recommendations, will be updated and modified in future editions of the JNC guidelines. In fact, to some extent the discussion in 2014 does not fully apply to the situation in 2017 where SPRINT has shown benefits of more intensive blood pressure treatment also in the elderly [3]. One should also bear in mind that guidelines indeed provide general guidance but that treatment decisions in individual patients will always be driven by personspecific factors based on the clinical picture and experience of the physician. There is a fine balance between an abstract population benefit of tight blood pressure control (that can of course also translate to individual benefits) and the concrete adverse effects in individual patients including dizziness, falls risk, risk of renal failure and electrolyte disturbance, and generally polypharmacy - particularly in the elderly. However, this concept is not specific to the elderly, and treatment decisions will always be tailored to the individual with guidelines providing, indeed, only guidance.

In this issue of the Journal of Hypertension we find further data on blood pressure targets in the elderly in an article by Nayor et al. [10]. The authors have modelled in two large general population cohorts, the Framingham (FHS) and Jackson Heart Studies (JHS), the incidence of cardiovascular events in people above the age of 60 years with blood pressure (treated or untreated) in the range of 140-149 $\mathrm{mmHg}$ systolic and $<90 \mathrm{mmHg}$ diastolic. Compared to people without hypertension their risk was consistently higher, and this risk extended to those aged 60 years and older. The authors conclude that treatment to JNC8 recommendations in the elderly, aiming at blood pressure below $150 \mathrm{mmHg}$ systolic, is associated with substantial residual risk of cardiovascular events.

Such epidemiological data have been available for many years. For example, the Prospective Studies Collaboration showed direct relationships between blood pressure and cardiovascular risk across all ages and levels of blood pressure [11] and there are other largescale population studies that have fuelled the discussions on blood pressure targets in recent years. In fact, an analysis by Bavishi et al. [12] along the same lines as the data provided by Nayor et al. arrived at very similar conclusions. Such data will always have to be interpreted 
with caution as they do not derive from randomised controlled trials but they can inform the discussion. In this sense the data by Nayor et al. [10] are important albeit probably not surprising. They have, however, been derived from contemporary US American population cohorts and should have particular relevance to the JNC that in the first instance addresses the situation in the USA.

We recommend the reader to critically assess the evidence provided in the article by Nayor et al. [10]. There are strengths and weaknesses but in the light of decades of discussions on blood pressure targets it cannot be the task of this editorial to add further arguments. The data in the article by Nayor et al. are clear and well presented and appear to support the notion that $150 / 90 \mathrm{mmHg}$ is too high a threshold for initiation of antihypertensive therapy and too high a treatment target in the elderly.

A few issues in the article by Nayor et al. [10], however, deserve special attention. First, the data derive from two cohorts with different blood pressure measurement protocols. We have seen in the post-SPRINT discussions how important the exact measurement protocols can be; depending on the method used the actual measured blood pressure can differ by several, maybe up to $10 \mathrm{mmHg}$. Even tiny blood pressure differences only occurring during certain phases of a trial can result in differences in cardiovascular outcomes as the Valsartan Antihypertensive Long-term Use Evaluation (VALUE) trial has painfully demonstrated [13]. We do not know if the FHS and JHS data can be meaningfully combined, even if the metaanalysis approach and the provided sensitivity analyses offer some reassurance. Second, not much is known about the details of antihypertensive therapy in the FHS and JHS participants - when was the therapy initiated, why was it initiated, what targets were defined for individual patients by their physician, what (e.g. adverse effects) has driven a change in therapy? It should also be noted that blood pressure targets will have generally changed over decades in parallel to updated guidelines. All this information would be important beyond the simple numerical blood pressure values that have been used for the present analysis by Nayor et al. [10]. And third, this analysis has been performed with the benefit of hindsight but not as a prospective clinical trial. It is somewhat easier to explore existing datasets than to set up a prospective clinical trial to more robustly answer a clinical question, and as with every secondary or not pre-defined analysis there is a risk of both false positive and false negative findings.

We would like to leave the exact interpretation of the data by Nayor et al. [10] to the clinical community and to future guideline committees. Instead we would like to put this paper into the current precision medicine landscape and see it as a wake-up call for the hypertension community to generate better and more detailed data [14,15]. As valid as the analysis of a relationship between blood pressure level and outcome may be on the population level or in the context of large clinical trials, the treatment of individual patients often requires information beyond such simple data. We have already mentioned such factors including duration of hypertension, adverse effects, degree of organ damage and comorbidities above, and they can be supplemented by biomarkers, genetic and genomic information and other factors that stratify patients into specific groups with regard to risk and treatment options. In fact, this information has the potential to define individual subtypes of hypertension and 
characterise the phenotype far better than the numbers that we read on a sphygmomanometer.

Such information is not available in the present analysis of FHS and JHS data by Nayor et al. [10] and in all fairness, is not available in any of the larger population based and clinical trial datasets. The best we can currently do is indeed what Nayor and colleagues have done: to analyse blood pressure figures against defined outcomes, adjusted for the available relevant clinical data. We thereby reduce a complex phenotype to a readout and miss opportunities to define tailored treatments for individual patients or patient groups. In the case of the elderly this is not only a question of missing molecular (biomarker, genetics) data but very much also a question of stratification by sex where one would expect differences in the pathophysiological make-up of hypertension in postmenopausal women who "catch up" with their cardiovascular risk compared to men who have different onset and development of high blood pressure. By simply adjusting for sex we cannot answer such fundamental questions in existing datasets.

In summary the paper by Nayor et al. [10] provides data that inform the discussion on blood pressure targets but even more importantly, it provides food for thought for the design of future clinical trials and general population cohorts. The experienced team of Nayor and colleagues have carefully analysed the available datasets using sophisticated statistical methods and are still limited by the missing phenotypic depth of these datasets. If we really want to enter the precision medicine age also for the diagnosis and treatment of hypertension we have to generate deeper phenotypic data and will then, hopefully, be able to answer questions about blood pressure targets and all the other controversies that the hypertension community has struggled to answer for so many years.

\section{Acknowledgements}

Sources of funding: Our work is supported by grants from the European Commission (Cooperative Research Projects "sysVASC" (603288), "HOMAGE" (305507) and "PRIORITY" (101813)) and the British Heart Foundation (Centre of Research Excellence Award RE/13/5/30177).

\section{References}

1. Mancia G, Fagard R, Narkiewicz K, Redón J, Zanchetti A, Böhm M, et al. 2013 ESH/ESC Guidelines for the management of arterial hypertension: the Task Force for the management of arterial hypertension of the European Society of Hypertension (ESH) and of the European Society of Cardiology (ESC). J Hypertens. 2013; 31:1281-1357. [PubMed: 23817082]

2. Hansson L, Zanchetti A, Carruthers SG, Dahlöf B, Elmfeldt D, Julius S, et al. Effects of intensive blood-pressure lowering and low-dose aspirin in patients with hypertension: principal results of the Hypertension Optimal Treatment (HOT) randomised trial. HOT Study Group. Lancet. 1998; 351:1755-1762. [PubMed: 9635947]

3. SPRINT Research Group. Wright JT Jr, Williamson JD, Whelton PK, Snyder JK, Sink KM, et al. A randomized trial of intensive versus standard blood-pressure control. N Engl J Med. 2015; 373:2103-2116. [PubMed: 26551272]

4. Kjeldsen SE, Narkiewicz K, Hedner T, Mancia G. The SPRINT study: outcome may be driven by difference in diuretic treatment demasking heart failure and study design may support systolic blood pressure target below $140 \mathrm{mmHg}$ rather than below $120 \mathrm{mmHg}$. Blood Press. 2016; 25:63-66. [PubMed: 26743157] 
5. James PA, Oparil S, Carter BL, Cushman WC, Dennison-Himmelfarb C, Handler J, et al. 2014 evidence-based guideline for the management of high blood pressure in adults: report from the panel members appointed to the Eighth Joint National Committee (JNC 8). JAMA. 2014; 311:507520. Erratum in: JAMA 2014; 311:1809. [PubMed: 24352797]

6. Wright JT Jr, Fine LJ, Lackland DT, Ogedegbe G, Dennison Himmelfarb CR. Evidence supporting a systolic blood pressure goal of less than $150 \mathrm{~mm} \mathrm{Hg}$ in patients aged 60 years or older: the minority view. Ann Intern Med. 2014; 160:499-503. [PubMed: 24424788]

7. Butt DA, Mamdani M, Austin PC, Tu K, Gomes T, Glazier RH. The risk of hip fracture after initiating antihypertensive drugs in the elderly. Arch Intern Med. 2012; 172:1739-1744. [PubMed: 23165923]

8. Smulyan H, Mookherjee S, Safar ME. The two faces of hypertension: role of aortic stiffness. J Am Soc Hypertens. 2016; 10:175-183. [PubMed: 26725014]

9. Beckett NS, Peters R, Fletcher AE, Staessen JA, Liu L, Dumitrascu D, et al. Treatment of hypertension in patients 80 years of age or older. N Engl J Med. 2008; 358:1887-1898. [PubMed: 18378519]

10. Nayor M, Stevenson MD, Musani SK, Xanthakis V, LaValley MP, Larson MG, et al. Incidence of cardiovascular disease in individuals affected by recent changes to US blood pressure treatment guidelines. J Hypertens. 2017

11. Lewington S, Clarke R, Qizilbash N, Peto R, Collins R. Prospective Studies Collaboration. Agespecific relevance of usual blood pressure to vascular mortality: a meta-analysis of individual data for one million adults in 61 prospective studies. Lancet. 2002; 360:1903-1913. Erratum in: Lancet. 2003; 361:1060. [PubMed: 12493255]

12. Bavishi C, Bangalore S, Messerli FH. Outcomes of intensive blood pressure lowering in older hypertensive patients. J Am Coll Cardiol. 2017; 69:486-493. [PubMed: 28153104]

13. Julius S, Kjeldsen SE, Weber M, Brunner HR, Ekman S, Hansson L, et al. Outcomes in hypertensive patients at high cardiovascular risk treated with regimens based on valsartan or amlodipine: the VALUE randomised trial. Lancet. 2004; 363:2022-2031. [PubMed: 15207952]

14. Dominiczak A, Delles C, Padmanabhan S. Genomics and precision medicine for clinicians and scientists in hypertension. Hypertension. 2017; 69:e10-e13. [PubMed: 28193712]

15. Currie G, Delles C. The future of "omics" in hypertension. Can J Cardiol. 2017; 33:601-610. [PubMed: 28161100] 